\title{
La construcción institucional del "loco peligroso""
}

\section{Institutional Construction of the "Dangerous Madman"}

Recibido: diciembre 19 de 2008 | Revisado: junio 10 de 2009 | Aceptado: junio 14 de 2009

\author{
OMAR Alejandro Bravo** \\ Universidad Nacional de Brasilia, Brasil
}

* Artículo de investigación

** Dirección postal: Iriondo 1877, CP 2000. RosarioArgentina.

Correo electrónico: omarlakd@gmail.com

\section{RESUMEN}

Los individuos que infringen la ley, considerados portadores de enfermedad mental y declarados legalmente inimputables, son recluidos en instituciones específicas. Para definir esa condición de inimputable es necesaria la realización de un laudo psiquiátrico que defina su patología y condición de peligroso. La relación que se produce aquí, entre el discurso psiquiátrico y el jurídico en asociación con determinadas prácticas institucionales, lleva al deterioro social e individual de estos sujetos y a su cronificación institucional. Esta investigación tuvo como objeto analizar las características de esos discursos y su funcionalidad institucional y social. Los laudos psiquiátricos de los reclusos del Ala de Tratamiento Psiquiátrico de la Prisión de Coméia, Brasil, constituyen la información sobre la cual se basó este análisis.

Palabras clave autor

Salud mental, prisión, psicología juridica, análisis de discurso, locura, violencia, subjetividad.

Palabras clave descriptor

Psicología forense, salud mental, análisis del discurso, presos.

\section{A B S T R A C T}

Individuals who infringe laws, considered mentally ill and declared legally immune from prosecution, are in some cases secluded in specific institutions. To define the condition of immune from prosecution it is necessary to make a psychiatric verdict which may also define his pathology and his condition of danger. This way, the relation between psychiatric discourse and judiciary discourse in association with certain institutional practices leads to the individual and social deterioration of these people and this process produces an institutional chronification. This research aims to analyze the characteristics of these discourses and their institutional and social functionality. The psychiatric verdicts are of recluse individuals in the Psychiatric Treatment Area of the Colméia Prison of Brasilia and constitute the main information of which this analysis bases on.

Key words author

Mental Health, Prison, Forensic Psychology, Discourse Analysis, Madness, Violence, Subjetivity.

Key words plus

Psychology Forensic, Mental Health, Discourse Analysis, Prisoners. 
En la época actual, con la afirmación de las políticas económicas neoliberales, contingentes enteros de la población mundial son expulsados del mercado de trabajo y privados del acceso a bienes materiales y simbólicos, siendo sometidos así a un creciente debilitamiento de sus vínculos sociales. Esto se expresa en un aumento de la violencia, de las enfermedades físicas y psíquicas, y en la fragilización de los lazos de solidaridad y resistencia.

Esta realidad genera también un aumento en los mecanismos institucionales de contención y estigmatización de esos sectores. Para el funcionamiento de estos mecanismos, cada sociedad organiza también modelos jurídicos y represivos que califican, rotulan y castigan determinadas conductas y personalidades. La perspectiva criminológica, conocida como labelling aproach, destaca cómo determinados procesos de estigmatización de grupos o personas considerados infractores de la ley, contribuyen a consolidar una identidad caracterizada por su condición marginal, otorgándole un cierto estatus social (Baratta, 2004).

Se coincide aquí con Bogo Chies (1997) en que el Estado, principalmente el capitalista, se objetiva a través de instituciones que actúan como fiadoras del sistema social vigente y operan como articuladoras y organizadoras de esa sociedad.

Así mismo, se generan instituciones y discursos que justifican estos aparatos y ocultan su sentido. Determinadas ciencias y sus prácticas tienen en este motivo no sólo la explicación de su funcionamiento, sino también la propia razón de su existencia, a pesar de que muchas veces exceden este propósito inicial. Foucault (1979) describe las dimensiones de esta articulación al mostrar como “...as práticas sociais podem chegar a engendrar dominios de saber que não somente fazem aparecer novos objetos, novos conceitos, novas técnicas, mas também fazem nascer formas totalmente novas de sujeitos e de sujeitos de conhecimento" (Foucault, 1979, p. 9).

La flexibilidad de estas redes institucionales y su carácter discursivo y práctico fluctúa fundamentalmente con relación al carácter y la cantidad de excluidos que el sistema provoca.
Wacquant (2001) relaciona la aparición de las políticas denominadas de "Tolerancia Cero" con la necesidad de estigmatizar y responsabilizar a determinados sectores sociales por sus condiciones de vida, colocando la necesidad de su punición y control como un imperativo relacionado con una cierta concepción de higiene social. La aplicación de estas políticas produjo un aumento enorme de la población carcelaria en los países en las que fueron aplicadas.

Una de sus bases teóricas es la doctrina de la "ventana rota" de Wilson y Kelling (1982) que afirma que un individuo que comienza rompiendo un vidrio, seguirá una carrera ascendiente en la escala de infracciones, si no es castigado con severidad por este primer hecho. El consumo de drogas ocupa un lugar destacado en esta progresión, siendo que este bárbaro e indemostrable argumento parece haber penetrado ciertas prácticas institucionales $\mathrm{y}$ discursos forenses.

De esta manera, determinadas técnicas y saberes operan al servicio de una concepción de derecho penal que, según Zaffaroni y Pierángeli (1999), es una

...construção destinada a cumprir certa função sobre algumas pessoas a respeito de outras..." sendo que "...sob determinadas condições histórico-sociais os sistemas penais selecionam um grupo dos setores mais humildes e marginalizados, os criminaliza e mostra ao resto dos setores marginalizados como limites do seu espaço social. (p. 14)

Verbitsky (2002) apunta la desigualdad en los ingresos como una de las causas estructurales del aumento de la violencia y de los crímenes contra la persona y la propiedad (inclusive siendo esta correlación aún más directa que la existente entre el índice de desempleo y el aumento de la criminalidad). En el Distrito Federal, Brasil, estas diferencias aparecen más acentuadas a partir de la separación espacial entre las ciudades satélites y la capital, Brasilia, ésta última con una población de mayor renta per cápita y mejor calidad de vida en general (IBGE, 2002). 
Las prisiones brasileñas muestran un mapa humano socialmente homogéneo, ya que en general sólo albergan personas de condición humilde, pero variada desde el punto de vista jurídico en relación con los motivos que provocaron la pena (Batista, 1999). Se incluyen aquí tanto a consumidores de drogas consideradas ilegales, como a aquellos sujetos que han realizado pequeños hurtos o que han cometido el delito en condiciones que merecerían un diagnóstico de inimputabilidad, según constató la II Caravana Nacional de Derechos Humanos realizada por la Comisión Nacional de Derechos Humanos, en las principales prisiones de Brasil en el año 2000.

Sobre esta última cuestión (el diagnóstico de inimputabilidad) se construyó buena parte de la relación entre el derecho penal y la psiquiatría. Este vínculo se inició desde el momento en que ésta comienza a definir su objeto de interés e intervención. Para Foucault (2001), esta definición de objeto proviene de la demanda de la propia justicia de comienzos de la época moderna que, a partir de la reforma propuesta por Beccaria (2000) en la segunda mitad del siglo XVIII y fundamentalmente en función de la nueva organización social en formación, precisa explicar y encuadrar las conductas de los sujetos infractores de la ley.

Antes de Beccaria, esta discriminación no era necesaria: el delito cometido era considerado una ofensa al soberano y debía ser castigado por una cuestión elemental de reparación que estaba más allá del sujeto infractor y sus motivaciones, para constituirse en una demostración de poder dirigida al conjunto de la sociedad en general.

Con la implantación del principio de personalización de las penas se abrió un espacio para considerar la posibilidad de que el hecho ilegal cometido obedeciera a una elección basada en el libre albedrío del sujeto o, por el contrario, si había acontecido en circunstancias o condiciones que permitiesen suponer la inimputabilidad del mismo, es decir, la falta de conciencia moral sobre sus actos.

Esta discriminación permite la implantación en los cuerpos penales de las denominadas medidas de seguridad. El Artículo 26 del Código Penal brasileño (1994) permite anular las penas de los sujetos considerados penalmente inimputables y substituirlas por otro tipo de medidas.

Dicho artículo estipula:

E isento de pena o agente que, por doença mental ou desenvolvimento mental incompleto ou retardado era, ao tempo da ação ou omissão, inteiramente incapaz de entender o caráter ilícito do ato ou de determinar-se de acordo com esse entendimento.

En Brasil, este tipo de medidas comienza a ser implementada en los años 40, con la importación y adaptación del denominado Código Rocco, de origen italiano. Históricamente, se confrontaron dos grandes tesis al respecto de la noción de enfermedad mental con posibilidad de ser vinculada a la condición de inimputable de quien la padece: la alienista, que entiende que solamente los psicóticos pueden ser declarados inimputables; y la nosológica, que amplia esta posibilidad a otras alteraciones mórbidas (toxicomanías graves, por ejemplo) (Rojas, 1932). Actualmente, esta segunda interpretación es la que goza de mayor consenso.

La seguridad a la que estas medidas se refieren es la del cuerpo social, de la población, que debe ser preservada de la convivencia social con sujetos irresponsables de sus actos. La primera definición de los grupos humanos que se enmarcan dentro de esta caracterización evidencia su espíritu: los anarquistas y los locos merecieron la misma preocupación por su control y separación del resto de la sociedad.

A finales del siglo XIX, Lombroso (1913) afirmó que era posible detectar la personalidad criminal a través de determinados rasgos físicos, como la forma del cráneo o las orejas, por ejemplo. La maldad del hombre "...estaria estampada no seu corpo" (Carrara, 1998, p. 101) y tendría un carácter atávico, definitivo, que permitiría retirar de la convivencia social a estos sujetos, quienes deberían, entonces, ser castigados por su peligrosidad, no ya por el hecho ilegal cometido.

Lombroso consolida así la aproximación entre psiquiatría y derecho penal (a pesar de que él se definiera como antropólogo criminal, son estas dos 
ciencias las que dan soporte histórico a su teoría). Este camino ya había comenzado a ser transitado con anterioridad por Pinel, Esquirol y Morel, entre otros (Zaffaroni \& Pierángeli, 1999). El propio Lombroso relativiza posteriormente sus afirmaciones al contemplar la influencia de factores ambientales y sociales en la aparición de conductas delictivas, como una forma de que su teoría fuese más aceptada en los ámbitos judiciales. En la actualidad, la antropología criminal no tiene apoyo ni en la psiquiatría ni en el derecho penal; sin embargo, la noción de un carácter atávico presente en el infractor de la ley aparece en algunas teorías y discursos, como las que procuran una base genética como explicación para los fenómenos violentos, por ejemplo.

\section{Definiendo el objeto de la investigación}

En el Ala de Tratamiento Psiquiátrico de la prisión de Coméia del Distrito Federal (ATP), Brasil, se encuentran los sujetos que en el momento de su condena o posteriormente, fueron considerados inimputables, según el artículo 26 del Código Penal brasileño ya citado, siendo anulada su pena y sustituida legalmente por las denominadas medidas de seguridad, con indicación de reclusión y tratamiento especializados.

Los artículos 96, 97, 98 y 99 del Código Penal prevén también la posibilidad de que el tratamiento sea realizado de forma ambulatoria o a través de la internación del agente en establecimientos hospitalarios.

Esta ala psiquiátrica presenta una gran precariedad estructural. El lugar destinado a los internos es pequeño, frío y húmedo y no existen formas de entretenimiento u opciones de trabajo disponibles. El único desplazamiento permitido, el "baño de sol", consiste en un una hora diaria de acceso al patio interno del sector.

Allí se encontraban detenidos, hasta septiembre del 2004, sesenta y cuatro sujetos, con edades que variaban entre los veinte y los sesenta y siete años. Algunos se encontraban en esa condición desde hacía más de veinticinco años.
Cabe destacar que esta ala psiquiátrica es exclusivamente masculina; la única mujer presa considerada inimputable, comparte un espacio común con el resto de las detenidas, lo que genera el reclamo constante de las mismas, dadas las dificultades para la convivencia que motiva el avanzado grado de compromiso psíquico de esta interna.

Esta población no tenía en esa época acompañamiento profesional ni atención especializada de ningún tipo. Como mera forma de contención, dos presos comunes convivían con ellos, administrando la medicación psiquiátrica que una psiquiatra les facilitaba y que éstos distribuían según su propio y dudoso criterio.

Las medidas de seguridad exigen la realización de un laudo como máximo cada tres años. Estos laudos deben certificar el cese de la condición de peligroso del sujeto como primer paso para que éste recobre su libertad. Para tal fin, debe contar también con parientes dispuestos a recibirlo, también según el artículo 26 del Código Penal.

Estos exámenes son realizados, en el Distrito Federal, por psiquiatras del Instituto Médico Legal a través de entrevistas y, ocasionalmente, la aplicación de instrumentos psicotécnicos. La mayoría de los internos del ala psiquiátrica no consigue pasar estos exámenes y pocos mantienen contacto con sus familias (entre otros motivos, porque muchos de ellos atentaron contra miembros de su propio entorno familiar).

Según lo que mostró una lectura preliminar de estos laudos, dictámenes periciales parecían haber sido hechos en un único y rápido contacto con los sujetos, lo que permite poner en duda el grado de veracidad y afirmación de contenidos.

En las varias visitas realizadas, fue posible constatar un severo cuadro de deterioro físico y psíquico de la gran mayoría de los internos de esta Ala. Los que presentaban un compromiso psíquico mayor eran objeto de todo tipo de abusos por parte de los otros internos. La mayoría se comunicaba con extrema dificultad y parecía estar permanentemente sobre los efectos de altas dosis de medicación psiquiátrica.

Dada las inquietudes e interrogantes suscitados por la descripción de esta realidad, se determinaron 
como objeto de esta investigación -que formó parte de la tesis de doctorado del autor- las instituciones, prácticas y discursos que intervienen en la determinación de la inimputabilidad penal y sanción de reclusión en instituciones especializadas de determinados sujetos infractores de la ley. Se consideró, en especial, la forma como esos mecanismos operan en el ámbito particular del Distrito Federal de Brasil y con relación a los sujetos que cumplen medidas de seguridad en el Ala de Tratamiento Psiquiátrico de la prisión de Coméia. Se situó así en el centro del análisis, al funcionamiento del sistema penal y su relación con las prácticas, saberes y discursos que lo atraviesan, entre ellas la psiquiatría, que ocupa un lugar fundamental en este proceso.

\section{Metodología}

La metodología utilizada para el análisis de la información fue de carácter cualitativo, donde el hecho empírico "...cobra su sentido en el marco de las construcciones deductivas y/o configurativas dentro de la teoría" (González Rey, 1997, p. 15).

Según Morin (1990), el método es la actividad reorganizadora necesaria para la teoría. El conocimiento influye en la construcción teórica, que no opera aquí como un punto fijo e invariable de referencia.

Las herramientas metodológicas empleadas fueron el Análisis de Discurso y el Análisis Institucional. El primero reúne diferentes áreas de conocimiento: el materialismo histórico, como teoría de las formaciones sociales y sus transformaciones, principalmente a partir de Althusser (1974); la lingüística de Saussure (1967) como teoría de los mecanismos sintácticos y de los procesos de enunciación; y la noción de discurso, basada principalmente en la producción de Foucault (1973) como teoría de la determinación histórica de procesos semánticos (Bravo, 2000; Pêcheux \& Fuchs, 1975). Esta articulación se produce a partir de tres operadores teóricos principales: la formación social, la lengua y el discurso, atravesadas por una noción de subjetividad de base psicoanalítica.
La corriente inglesa del Análisis de Discurso, desarrollada principalmente por Fairclough (1989) y Thompson (1984) permite superar el carácter estructuralista inicial de esta teoría a través del reconocimiento del carácter activo del sujeto en la producción del discurso y sus modificaciones y de la dimensión intertextual de los discursos y textos, por estar atravesados por fragmentos de otros discursos que se manifiestan de forma parcial o velada. Determinados discursos se tornan hegemónicos cuando se afirman como sentido común, lo que permite naturalizar sus contenidos.

La escuela inglesa define tres etapas o momentos analíticos: el de descripción, que trabaja sobre la base sintáctica de los textos o discursos; el de interpretación, que determina el tipo de texto y su relación con otros textos o discursos; y, el de explicación, que trabaja sobre las determinaciones de poder que el discurso, como práctica social, establece en las instituciones. El discurso se relaciona aquí con una práctica social de la cual él es constituido y constituyente. Se analizan las relaciones de poder e ideológicas en los niveles situacionales, institucionales y sociales.

En esta investigación fueron consideradas solamente las dos últimas etapas de análisis. La primera, que se basa principalmente en la estructura sintáctica de los textos, fue excluida por ser más adecuada al análisis de diálogos y entrevistas, en que operadores verbales y modos discursivos toman determinados sentidos ideológicos e interrelacionales específicos.

Los discursos considerados significan y se refieren a prácticas institucionales dentro de las instituciones analizadas. Por este motivo, el Análisis Institucional operó como complemento metodológico obligado de esta investigación.

El Análisis Institucional se propone analizar e intervenir en los conjuntos sociales con el propósito de mejorar la calidad de vida de las personas, contando con la participación activa de éstas para esta tarea. Guattari y Deleuze (1976) afirman que esa dirección de análisis no es sólo de un individuo o grupo, sino de un conjunto de procesos sociales. De esta manera, se mantiene una dirección de 
intervención dirigida a la promoción de la salud mental en determinados grupos y conjuntos humanos.

Se entiende aquí que el objeto de la salud mental "... no es de modo exclusivo el individuo o los conjuntos sociales, sino las relaciones que permiten pensar conjuntamente al individuo y su comunidad" (Galende, 1997, p. 31). Este abordaje permite que las intervenciones incluyan como objetivos prioritarios, la integración social y comunitaria de los sujetos, promoviendo redes sociales de contención que posibiliten tramitar situaciones de violencia, desamparo y sufrimiento. Esa visión amplia permite evitar también los riesgos de determinar grupos de anómalos sociales (adolescentes, usuarios de drogas, infractores de la ley, locos) que deban ser tutelados o controlados.

Guilhon (1980) establece tres planos sucesivos de análisis en las instituciones: el de las prácticas, que se articulan en aparatos (entendidos como un conjunto de prácticas estructuradas) que, a su vez, constituyen formaciones. Cada uno de estos planos puede ser considerado en relación con sus aspectos económicos, ideológicos y políticos.

La disputa o tensión entre los discursos dominantes y los que intentan disputar esa hegemonía corresponden a lo que el Análisis Institucional entiende como un conflicto entre lo instituido y lo instituyente ${ }^{1}$.

Para Lourau, lo instituyente está relacionado a la capacidad de innovación, de contestación, y lo instituído al "...orden establecido, los valores, los modos de representación considerados normales como igualmente los procedimientos habituales de previsión" (Lourau, 2004 citado por Altoé, 2004, p. 22).

En el contexto particular de esta investigación, se entiende que lo instituido está representado, por una parte, por el sistema penal en su totalidad, considerado como el control social punitivo institucionalizado (Zaffaroni \& Pierángeli, 1999), y compuesto de tres segmentos básicos: el policial,

1 El instituido, resultado de la acción instituyente, tiene una tendencia a permanecer estático y conservador. El instituyente es el proceso de cambio de lo instituido (Baremblitt, 1992). el judicial y el ejecutivo; y, por otra parte, por la psiquiatría, como ciencia y discurso del tratamiento de la locura basada principalmente en la exclusión social del enfermo mental, mecanismo éste cuyo contenido sancionatorio la aproxima a la definición y características del sistema penal.

Fueron considerados, como corpus del análisis, sobre un total de 64 , los laudos presentes en los 48 procesos penales de los internos que cumplían medida de seguridad en el Ala de Tratamiento Psiquiátrico de la Prisión de Coméia. Otros procesos no se encontraban disponibles por diversos motivos (trámites en otros juzgados, por ejemplo).

En el análisis de la información recolectada fue considerado de relevancia:

1. El diagnóstico de peligrosidad, atendiendo a la definición de la misma como potencial, actual o latente.

2. Las categorías nosográficas utilizadas y la relación entre las mismas.

3. La relación entre tipo de crimen, categorías nosográficas y diagnóstico de peligrosidad.

En el análisis de la información, se escogieron tres casos por ser considerados paradigmáticos en relación con el funcionamiento de los mecanismos institucionales analizados. A ellos se suman, con el propósito complementario de establecer una lectura histórica de estos discursos y prácticas, algunos laudos producidos en el Manicomio Judicial Heitor Carrilho de Río de Janeiro, entre los años 1933 y 1959.

\section{Análisis de la información}

\section{Peligroso por falta de familia (B)}

B., de sesenta y cuatro años, y su familia llegaron de la región nordeste del país a Sobradinho, ciudad donde vivían en la época del crimen. En el año 1966, B. agredió a una vecina con un cuchillo, sin motivo aparente, causándole graves heridas. Según los laudos, ya presentaba antecedentes de internaciones psiquiátricas en los años de 1964 y 1966. 
Ingresado en la prisión de Brasilia, en ese mismo año, fue sometido a la realización de un dictamen psiquiátrico, dado el comportamiento bizarro que mostraba en la prisión. En este primer laudo se consideró al inculpado como peligroso para la comunidad carcelaria, siendo diagnosticado como débil mental. En el final del mismo texto, aparece otro diagnóstico diferente al primero: el de esquizofrenia. El laudo siguiente es bien posterior, del año de 1979, y fue realizado en el Manicomio Judicial Heitor Carrilho de Río de Janeiro, sin que conste en el proceso la causa o la época de la transferencia. B. es considerado aquí como peligroso, con un diagnóstico de esquizofrenia paranoide.

Del Heitor Carrilho, B. pasó al Hospital Psiquiátrico de Anápolis y de aquí al de Paranoá, donde vivía su hermano, registrando también un período de internación en la Clínica São Miguel. En 1994, recibe autorización para una salida temporal, y se va a vivir con su padre en el Estado de Piauí. Con él comenzó a trabajar como pescador, aparentemente sin presentar problemas de conducta o de salud. A pesar de esto, y por el hecho de haber violado las condiciones de su régimen legal (no se presentó en el período previsto), es requerido nuevamente por la justicia, debiendo volver al Ala de Tratamiento Psiquiátrico.

El laudo siguiente, del año 1995, diagnostica esquizofrenia terminal, sin cesación de peligrosidad. El dictamen del año 1996 mantiene este diagnóstico, aclarando que aún es considerado peligroso "... por falta de familia dispuesta a recibirlo”. Dos años después, en otro laudo, la conducta del periciado es considerada excelente, así como su relación con las autoridades y los compañeros, siendo ratificada su condición de esquizofrénico.

El enunciado de peligrosidad de $B$. es relacionado, en primera instancia, con una patología psiquiátrica. Más tarde, esa condición de peligroso se mantiene, pero ahora en función de la falta de familiares que pudiesen recibirlo. La funcionalidad y plasticidad institucional de este enunciado aparece aquí claramente: el diagnóstico de peligrosidad sirve para un propósito administrativo, relacionado con la exigencia de un soporte familiar para poder otorgar salidas temporales y altas definitivas a los sujetos que cumplen medidas de seguridad.

Paradójicamente, $B$. había pasado un tiempo con un familiar, mostrando condiciones para el reintegro a una vida social plena. La justicia, absurdamente, le exigió su retorno al Ala de Tratamiento Psiquiátrico, para después exigirle el mismo vínculo del cual se lo había privado.

Es posible ver aquí como el diagnóstico de peligrosidad no mantiene una lógica de asociación con determinadas categorías clínicas o conductas, sino que opera con un propósito jurídico-institucional. El enunciado de peligrosidad, definido y operacionalizado por el discurso médico-psiquiátrico, se inscribe aquí en un discurso más amplio de carácter jurídico-burocrático.

\section{El peligroso latente (D)}

D., de 43 años de edad, vivía en una ciudad satélite de Brasilia en la época en que cometió el crimen que lo llevó a la ATP. Según su proceso, no consiguió completar el ciclo primario debido a frecuentes crisis de nervios. Trabajó por poco tiempo en el Servicio de Limpieza Urbana; después, de forma independiente, en trabajos ocasionales.

En el año 1985, en estado de ebriedad, D. asesinó a su hijo de tres años con una barra de hierro. Antes de cometer ese acto, había ido a la policía a denunciar que cometería un crimen de ese tipo, denuncia que no fue atendida. Originalmente, destinado a prisión común, agredió sin motivo a un agente penitenciario, según los laudos. En la época del crimen, tenía dificultades para dormir y manifestaba su voluntad de cometer suicidio. Su familia no lo visitaba.

El primer laudo, del año 1985, certifica a D. epiléptico, citando crisis que comenzaron a los ocho años de edad. Destaca también la historia familiar: el casamiento posterior a la muerte del padre, las peleas frecuentes con su esposa y la prohibición de los suegros de que la hija lo acompañase a trabajar en otro Estado, lo que habría desencadenado la crisis que lo llevó a cometer el crimen.

En este examen se consideró que el crimen fue cometido en situación de estado crepuscular 
—definida por Ey (1978) como el momento en el cual se pierde la conciencia de los actos realizados-. El diagnóstico fue el de personalidad epileptoide con inestabilidad emocional, sin autocontrol y violento en las relaciones interpersonales, con poco grado de recuperación. Por todo esto, $D$. es considerado peligroso en alto grado.

El siguiente dictamen es del año 1992. El estado del paciente parece haber empeorado, sufriendo ahora de delirios persecutorios y convulsiones frecuentes. Considerado nuevamente como peligroso, este texto termina con una conclusión sorprendente: aún no existen sistemas penitenciarios que puedan dar cuenta de casos como éste.

El laudo del año 1993 cambia la caracterización clínica: $D$. es diagnosticado ahora como portador de esquizofrenia residual, sociopatía y oligofrenia, diagnósticos estos incompatibles entre sí.

Los dos dictámenes siguientes, realizados, en los años de 1993 y 1994, apenas ratifican la necesidad de la continuidad de D. en la ATP. En el de 1996 se destaca su progreso, encontrándose el paciente trabajando normalmente en el penal y manteniendo una buena relación con las autoridades y los otros internos. No obstante, según el texto, se consideró que mantenía su peligrosidad en estado latente, estando la misma inhibida por la rutina carcelaria.

En 1997 es nuevamente considerado peligroso, asociada esa condición al diagnóstico de epilepsia esquizoide. Apenas en el examen realizado en el año 1998, y frente a la inminente apertura de una Casa Abrigo destinada a población psiquiátrica, se recomienda tratamiento. D. había acumulado, en la época de esta recomendación, 13 años de reclusión en la ATP.

Sufriendo ya de un notable deterioro físico y psíquico, se considera en el dictamen de 1998, que el sujeto tendría su peligrosidad cesada desde que tuviese acompañamiento clínico y familiar. Los laudos posteriores autorizaron salidas temporales, que se cumplieron con frecuencia irregular.

El caso de D. reúne casi todas las formas posibles de diagnóstico de peligrosidad: peligroso en alto grado, peligrosidad en estado latente, peligroso (sin otras consideraciones) y peligrosidad cesada. Estas caracterizaciones se suceden sin un orden lógico aparente, ya que cambian de un laudo para otro sin un motivo o asociación definida con otros indicadores. De la peligrosidad latente del año 1996, se pasa nuevamente a la condición de peligroso al año siguiente, y a la cesación de peligrosidad en el posterior.

Los diagnósticos clínicos son diferentes y hasta contradictorios. Epiléptico en un primer momento, D. pasa a ser esquizofrénico, posteriormente (probablemente, por manifestar delirios) siendo también, y en un mismo laudo, considerado sociópata y oligofrénico.

Nuevamente, estos diagnósticos operan apenas como complemento de la sanción jurídicomédica de peligrosidad. El enunciado presente en el laudo del año 1992, que refiere a la inexistencia de sistemas penales capaces de alojar a este tipo de sujetos, se inscribe en un discurso histórico de la psiquiatría, de exigencia permanente de creación de nuevos sistemas de reclusión para poblaciones cada vez más específicas. Llama la atención aún más que la demanda sea por sistemas penitenciarios y no terapéuticos, como serían en teoría la ATP y los manicomios judiciales.

El mismo tipo de caracterización de ese espacio institucional aparece en el enunciado que define a la rutina carcelaria como un inhibidor de la peligrosidad del sujeto. Rutina carcelaria, ni siquiera manicomial, que acabó inhibiendo no sólo su peligrosidad, sino también su condición humana, en general. Ese deterioro es lo que permitió la autorización para salidas temporales, que no fueron producto de un progreso clínico.

En la novela de Zola, La bestia humana (1885/1969), el protagonista sufre el impulso irrefrenable de cometer un crimen. En la lucha contra esa compulsión deja señales de su propósito, con la esperanza de ser detenido. De la misma forma, D. intentó llamar la atención sobre el crimen que finalmente cometió, más no tuvo otra institución diferente de la Policía adonde dirigir su angustia, que se tradujo fatalmente en el paso al acto que lo llevó a la ATP.

Tavolaro (2002) destacó, en un estudio hecho en el Manicomio Judicial Franco da Rocha, el hecho de que el $52 \%$ de los internos en esa institución 
habían buscado ayuda en instituciones públicas antes de cometer el hecho que los llevó a juicio, no siendo tampoco atendidos.

\section{Peligroso por rebelde $(\mathrm{F})$}

F. tenía 40 años de edad en la época en que fue hecha esta investigación. Nacido en el nordeste, se mudó al Distrito Federal cuando era niño junto con su familia. Comenzó a trabajar a los 12 años de edad casi siempre en empleos informales, no habiendo completado el ciclo primario de educación. F. era soltero y tenía un hijo al que no conocía. Su padre, alcohólico, había fallecido hacía mucho tiempo, su madre y hermanas vivían aún en el DF. Fue condenado por robo seguido de muerte en 1986, año en que invadió la casa donde trabajaba su pareja y junto con ella amordazaron a la empleada que trabajaba allí, que murió asfixiada. En 1991, recibió libertad condicional cuyos términos no cumplió, lo que lo llevó de vuelta a la prisión.

En 1997, ya en régimen ambulatorio, volvió a consumir drogas, lo que le provocó problemas con su familia. Aún en semilibertad, integró un grupo que robaba carros dirigido por su propio abogado, hecho por el cual fue nuevamente preso. Ya en prisión comenzó, según el laudo, a mostrar algunos comportamientos bizarros, agrediendo a un agente penitenciario. Por ese motivo fue derivado a la ATP.

F. pasó por muchos dictámenes en su extensa trayectoria psiquiátrico-penal. En el primero, hecho en el año 1986, relató que escuchaba voces. El psiquiatra consideró que esta manifestación clínica fue inventada por el periciado considerándolo, aún así, portador de disturbios psiquiátricos graves, dependiente de la marihuana y peligroso. El laudo siguiente, del año 1989, lo considera egocéntrico e inseguro y lo diagnostica como obsesivo compulsivo, recomendando salidas temporales. En el año 1994, en un nuevo laudo, F. es considerado portador de esquizofrenia hebefrénica y peligroso, destacándose, entre sus antecedentes, el uso prolongado de drogas. En el laudo de 1996, el diagnóstico cambia, pasando a ser el de trastorno de personalidad con predominio de manifestaciones psicopáticas.

El laudo de 1997 se refiere a un examen toxicológico obligatorio, que dio resultado negativo. A pesar de mantener el diagnóstico de sociopatía, este examen considera cesada la peligrosidad, desde que el periciado tenga apoyo de su familia para salidas parciales.

El dictamen de 1998 considera a F. nuevamente peligroso e impulsivo, no recomendando salidas temporales. La explicación para este cambio aparece destacada en el texto: se negó a tomar la medicación indicada por la psiquiatra por suponer que también era droga, evidenciando así, según el perito, su dificultad de lidiar con la realidad de forma satisfactoria. En el año 2000 es ratificada su peligrosidad, siendo declarado portador de trastorno de personalidad. El diagnóstico de peligrosidad fue ratificado en el laudo del año 2002, aclarando que este carácter se relaciona con su falta de empeño escolar y laboral.

El caso de F. es revelador de algunas características de los mecanismos discursivos que operan en estos laudos y en el discurso psiquiátrico en general. Nuevamente, existe aquí una falta de coherencia entre los sucesivos diagnósticos, que cambian de una figura patológica a otra. El discurso psiquiátrico consideró aquí como factor revelador de la peligrosidad de F. su negativa a seguir tomando medicación, asociada por el perito a su falta de adecuación a la realidad y, más tarde, a la falta de deseos de trabajar y estudiar.

Esta negativa, de una lucidez notable, es respondida por el discurso psiquiátrico con una doble condena: la de reclusión y la de medicación forzada. Algunas contradicciones pueden ser aquí destacadas: considerado dependiente de drogas y habiendo sido objeto hasta de exámenes toxicológicos obligatorios por ese motivo, F. muestra un cierto progreso clínico en ese sentido al negarse a consumir las drogas que más lo perjudicaban en ese momento: las administradas en la ATP. El discurso psiquiátrico parece no poder establecer otra dimensión posible, para los sujetos considerados inimputables, que no sea la de la peligrosidad o la del deterioro psíquico, factor éste que parece 
permitir el fin de aquella. Cuando $F$. articula una demanda que se enfrenta al criterio de verdad absoluta que la psiquiatría coloca en juego en estos exámenes, ésta lo sanciona, intentando anular así la denuncia e impidiendo un posible progreso del individuo.

El periciado es también obligado a demostrar interés en trabajar y estudiar, siendo exigida así una intención de recuperación y reinserción social que, por otra parte, es considerada como una manifestación patológica cuando se expresa en una dimensión crítica de la autoridad del discurso psiquiátrico.

F., quizás culpable del pecado de creer que los pájaros pueden dispararle a las escopetas, tomaba, en la época del último examen y según los laudos, haldol, fenergán, prometazina, carbamazepina e amitriptilina. F. era también portador del virus del $\mathrm{VIH}$, no recibiendo la medicación necesaria ni cuidados particulares.

\section{Otros discursos, una misma práctica: el Manicomio Judicial H. Carrilho de Río de Janeiro}

El Manicomio Judicial de Río de Janeiro tuvo como propósito, según su propio fundador y primer director H. Carrilho, ser el lugar de secuestro de los terribles, los incorregibles, los amorales constitucionales, para ofrecer a esa población la "...ortopedia moral de que carecen” (Carrilho, 1931, p. 30).

De uno de esos sujetos, el perito opinaba que "... su fisonomía refleja de algún modo su simplicidad mental, que recuerda, hasta cierto punto, a la de los individuos del campo" (Carrilho, 1930, p. 107). Esa consideración por el origen social del periciado aparece también en otros laudos, referida a la preocupación de un perito por el efecto que un libro como la Biblia podría causar "...en individuos incultos, destituidos de sentido crítico" (p. 153).

En un laudo del año 1934, el perito destaca la ineficacia de las penas aplicadas a aquel tipo de sujeto que "...vive en constante repugnancia al esfuerzo" (Carrilho, 1934, p. 23), merecedor por eso de medidas de seguridad. En el año 1937, el doctor
Bourgoy de Mendonça define como propósito de la psiquiatría la profilaxis de la criminalidad, y destaca la necesidad de actuar contra "...los vagabundos de origen étnico, económico-social y patológico” (Bourgoy de Mendonça, 1937, p. 15). Por eso, Nobre de Mello (1937) considera necesario que el "...delincuente sea encarcelado, no por lo que ha hecho, sino por lo que es" (p. 30).

Dos años después, un artículo que intenta profundizar la cuestión de la responsabilidad criminal se preocupa por "...recordar que un indio bárbaro puede ser inteligente" (Carrilho, 1941, p. 86). Otros periciados no merecen tantas consideraciones, ya que los exámenes verifican que "...su hábitat natural es la escoria y sus compañeros son todos maleantes y agitadores" (p. 161).

Buscando la etiología de esas alteraciones morales, años más tarde, se hace una consideración sobre la relación entre tuberculosis y conducta delictiva, llegando a la conclusión de que el delincuente tuberculoso puede transformarse en "...un oportunista con el sentido moral afectado", siendo que esa enfermedad "...da una cierta perversidad al individuo”, observando también que en ellos “...el sentido religioso disminuye (Arruda, 1959, p. 27). Otras causas externas podían generar ese tipo de conducta, como, por ejemplo, el uso de marihuana, considerada una "...planta asesina que libera la agresividad natural del hombre” (p. 62).

\section{Conclusiones}

En los textos analizados, la declaración de peligrosidad del infractor de la ley considerado penalmente inimputable, no se basa en criterios clínicos definidos, siendo que los diagnósticos son a menudo contradictorios entre sí y están permanentemente atravesados por otros discursos de carácter moralista o jurídico. La suposición intrínseca de una relación directa entre enfermedad mental y potencial delictivo parece sustentar este entramado discursivo. Esto se verifica en la comparación entre los discursos presentes en los laudos del Manicomio Judicial Heitor Carrilho y los del Ala de Tratamiento Psiquiátrico: los diagnósticos cambian, 
de acuerdo también a los modelos nosográficos de cada época; el carácter jurídico de los discursos, dirigidos a sancionar la supuesta asociación entre locura y peligrosidad con medidas de reclusión, se mantiene.

En estos textos, las cuestiones sociales e institucionales que rodeaban tanto a los hechos que llevaron a los sujetos a esa situación penal como a las consecuencias de esos procesos institucionales, aparecen como relegadas o desconocidas. La supuesta asepsia de los discursos presentes en los laudos les permite tomar distancia de las consecuencias institucionales que provocan en los sujetos que son objeto de los mismos.

El deterioro progresivo de la población que cumple medidas en la ATP es asociado a su patología. Por otra parte, los procesos de cura y reinserción social parecen depender únicamente de los efectos de su condición de reclusión.

El carácter ideológico de esos discursos aparece de forma más evidente en las consideraciones presentes en varios laudos sobre la relación entre el origen social de los sujetos, su nivel de inteligencia y su disposición para el trabajo, por ejemplo.

Dentro de la distinción hecha por Guilhon (1980) entre aparatos de reproducción material que responden a efectos económicos, aparatos de reproducción social con efectos predominantemente políticos y aparatos de reproducción imaginaria con efectos ideológicos, los mecanismos psiquiátrico-legales que se articulan a través de los laudos y las prácticas de reclusión asociadas, se encuadran en un tipo de aparato de reproducción imaginaria que funciona reproduciendo la supuesta asociación entre locura, pobreza y peligrosidad. Este aparato ideológico abarca también algunas prácticas secundarias de carácter político, como las representadas por la burocracia carcelaria. Esto contribuye a producir una doble identidad de los sujetos que cumplen medidas de seguridad: como locos y como presos, dentro de un dispositivo institucional que reúne lo peor de las prisiones con lo más aberrante de los manicomios.

De esta forma, estos sujetos no tienen condiciones de cualquier elaboración simbólica relacionada con el hecho cometido. El filósofo francés Althusser
(1992) después de haber asesinado a su esposa, fue declarado inimputable y sujeto a una medida de seguridad. En su obra El porvenir es largo, intentó elaborar de alguna forma su acto, elaboración ésta imposibilitada por su situación institucional, ya que afirma: "...es bajo la losa sepulcral del no ha lugar, del silencio y de la muerte pública bajo la que me he visto obligado a sobrevivir y a aprender a vivir" (p. 43). Lo que Althusser demanda es una inscripción en un registro simbólico que le permita evitar la condición de desaparecido (según su propia definición) que el confinamiento psiquiátrico le impuso.

De esta forma, las medidas de seguridad conducen al "loco infractor" a la permanencia por tiempo indefinido en los manicomios judiciales, instituciones que son "... as mais feroces e cerradas do campo psiquiátrico” (Lobosque, 2001, p. 34).

La deconstrucción de los mitos que históricamente se han tejido en torno a la locura no es tarea fácil, mucho menos mudar el sentido discriminatorio e inhumano con el que muchas instituciones tratan a los sujetos más fragilizados psíquicamente. Cuando la locura aparece asociada a un acto criminal, estas tareas se hacen más difíciles, pero al mismo tiempo más urgentes.

Esto obliga a un desplazamiento desde el lugar incómodo y limitado al que la ley, en general, nos convoca (el del mero parecer técnico, subsidiario del proceso legal), al más amplio de la salud mental, donde deben considerarse también las condiciones otorgadas a los sujetos para su tratamiento y recuperación. Es preciso para esto, poder responder a algunas preguntas de la justicia con un discurso particular y propio que impida, por ejemplo, determinar la condición de peligroso de un sujeto infractor en una entrevista o a través de la aplicación estandarizada y burocrática de algún instrumento psicotécnico.

Esto no significa negar el valor de la pericia ni el de las prácticas forenses en general, sino dotar a las mismas de otras potencialidades, de otros espacios discursivos y de acción.

En Latinoamérica en particular, la expulsión del mercado de trabajo y la imposibilidad de acceso a capitales simbólicos, culturales y materiales de contingentes enteros de la población, así como el 
deterioro de las instituciones de salud y asistencia, obliga a considerar el lugar que nuestra práctica ocupa en dichos procesos y la posibilidad de ubicarla en el lugar ético que esta realidad demanda.

\section{Referencias}

Althusser, L. (1974). Ideologia e aparelhos ideológicos do Estado. Lisboa: Lisboa.

Althusser, L. (1992). El porvenir es largo. Barcelona: Ediciones Destino.

Altoé, S. (2004). René Lourau: analista institucional em tempo integral. São Paulo: Hucitec.

Arruda, E. (1959). O delinqüente tuberculoso. Arquivos Manicômio Judiciário, 19(2), 27-58.

Batista, N. (1999). Introdução crítica ao Direito Penal Brasileiro. Rio de Janeiro: Rievan.

Baratta, A. (2004). Criminología crítica y crítica del derecho penal. Buenos Aires: Siglo XXI.

Baremblitt, G. (1992). Compendio de análise Institucional. Rio de Janeiro: Rosa dos Tempos.

Beccaria, C. (2000). De los delitos y las penas. Bogotá: Temis.

Bogo Chies, L. (1997). Prisão e estado. Pelotas: EDUCAT.

Bourgoy de Mendonça, M. (1937). Aspectos médicolegais e sociais do problema da vadiagem. Arquivos Manicômio Judiciário, 17(1), 13-18.

Brasil. (1994). Código Penal. São Paulo: Saraiva.

Bravo, O. (2000). Discurso sobre drogas nas instituições do Distrito Federal. Disertación de Maestría en Psicología Clínica, Universidad de Brasilia, D.F., Brasil.

Câmara dos Deputados. (2000). II Caravana Nacional de Direitos Humanos: Uma amostra da realidade prisional brasileira. Brasilia: Coordenação de Publicações da Câmara dos Deputados.

Câmara dos Deputados. (2000). I Caravana Nacional de Direitos Humanos: Uma amostra da realidade manicomial brasileira. Brasilia: Coordenação de Publicações da Câmara dos Deputados.

Carrara, S. (1998). Casos e acasos: o crime de um certo Custodio e o surgimento do Manicômio Judiciário no Brasil. Cadernos do Instituto de Medicina Social da Universidade Federal do Rio de Janeiro, 73-107.
Carrilho, H. (1930). Delinqüentes internados no Manicômio Judiciário Heitor Carrilho. Arquivos Manicômio Judiciário, 1(1), 47-67.

Carrilho, H. (1931). Anthropologia Penitenciaria. Arquivos Manicômio Judiciário,2 (2), 11-20.

Carrilho, H. (1934). Em torno das formas mentais prolongadas da encephalite epidémica. Arquivos Manicômio Judiciário, 4(1), 5-26.

Carrilho, H. (1941). A responsabilidade criminal no novo Código Penal. Arquivos Manicômio Judiciário, $11(3), 78-139$.

Ey, H. (1978). Tratado de Psiquiatría. Barcelona: TorayMasson.

Fairclough, N. (1989). Language and power. Londres: Longman Group.

Foucault, M. (1973). El orden del discurso. Barcelona: Tusquets.

Foucault, M. (1979). A verdade e as formas jurídicas. Rio de Janeiro: PUC.

Foucault, M. (2001). Os anormais. São Paulo: Martins Fontes.

Galende, E. (1997). De um horizonte incierto. Psicoanálisis y salud mental en la sociedad actual. Buenos Aires: Paidós.

González Rey, F. (1997). Epistemología cualitativa y subjetividad. La Habana: Pueblo y Educación.

Guattari, F. \& Deleuze, G. (1976). Mil mesetas. Valencia: Pré-Textos.

Guilhon de Albuquerque, J. (1980). Instituição e poder: a análise concreta das relações de poder nas instituições. Rio de Janeiro: Paz e Terra.

Instituto Brasileiro de Geografia e Estatística. (2002). Síntese de indicadores 2002. Uma análise das condições de vida da população brasileira. Obtenido el 26 de mayo, 2002, de www.ibge.gov.br

Lobosque, A. (2001). Experiências da loucura. Rio de Janeiro: Garamond.

Lombroso, C. (1913). L'uomo allienato. Roma: Fratelli Bocca.

Morin, E. (1990). Introdução ao pensamento complexo. Lisboa: ESF Editéur.

Nobre de Mello, S. (1937). Personalidade esquizotímica com desvios éticos constitucionais. Arquivos Manicômio Judiciário, 17(1), 29-33.

Pêcheux, M. \& Fuchs, C. (1975). Hacia el Análisis Automático del discurso. Madrid: Gredos. 
Rojas, N. (1932). Psiquiatría Forense. Buenos Aires: El Ateneo.

Saussure, F. (1967). Curso de lingüística general. Madrid: Bruguera.

Tavolaro, D. (2002). A casa do delírio: reportagem no Manicomio Judiciário Franco da Rocha. São Paulo: SENAC.

Thompson, S. (1984). Studies in the theory of ideology. Cambridge: Polity Press.

Verbitsky, H. (2002, septiembre 12). Morir de pena. El País (España), p. 9.
Wacquant, L. (2001). As prisões da miséria. Rio de Janeiro: Zahar.

Wilson, Q. \& Kelling, G. (1982). Broken windows: The police and the neighborhood safety. Atlantic Monthly, marzo, 29-38.

Zaffaroni, E. \& Pierangeli, J. (1999). Manual de direito penal brasileiro. São Paulo: Revista dos Tribunais.

Zola, E. (1885/1969). A besta humana. Rio de Janeiro: Tecnoprint. 
